\title{
SICHERES LICHT
}

\section{Liebe Leserin, lieber Leser,}

es ist eigentlich erstaunlich, wie oft in den letzten Monaten Autos unterwegs waren, die eine defekte oder falsch eingestellte Lichtanlage hatten. Dies betrifft Pkw, Bus und Lkw gleichermaßen, und hier alle Formen der Außenbeleuchtung eines Fahrzeugs vom Scheinwerfer über die Rück- und Bremsleuchten bis zum Kennzeichenlämpchen. Denn eigentlich hätte doch jeder im Aktionsmonat Oktober vor der dunklen Jahreszeit die Chance gehabt, kostenlos seinen „Einäugigen“ beim Lichttest von Zentralverband Deutsches Kfz-Gewerbe (ZDK) und Deutscher Verkehrswacht (DVW) überprüfen zu lassen.

Fast schon resignierend klingt da aus Bonn die Titelzeile der Pressemitteilung der beiden Institutionen: „Keine Besserung in Sicht“. Denn selbst bei Fahrzeughaltern, die pflichtbewusst sind und ihren Pkw testen ließen, sei die Mängelquote leicht gestiegen. Insgesamt war bei 35,2 \% der in den circa 38.000 Betrieben der Kfz-Innungen überprüften Fahrzeuge die Beleuchtung fehlerhaft (Vorjahr 34,3\%). Scheinbar ist es schwer, das Licht selbst in Schuss zu halten.

Dabei ist doch „Sehen und gesehen werden" der wichtigste Aspekt im Straßenverkehr. Aber viele kümmern sich nicht darum, obwohl diese Nachlässigkeit auch anderen schaden kann. Wann geht ihnen ein Licht auf? Dieser leichtsinnige Umgang mit der Beleuchtung kann sich mittlerweile auch bei der Hauptuntersuchung rächen. Schon eine kaputte Lampe zählt als sicherheitsrelevanter Mangel, sodass die TÜV-Plakette nicht vergeben wird und der Wagen kostenpflichtig neu vorgeführt werden muss. Zuvor wurde dies nur als geringer Mangel eingestuft.

Laut TÜV-Report 2014 ist das Licht sogar schon bei der ersten HU-Prüfung nach drei Jahren mit einer Quote von $7 \%$ trauriger Mängel-Spitzenreiter - weit vor Öl- verlust (1,1\%) und Bremsanlage (1,0\%). Bei älteren Fahrzeugen verschärft sich dieser Trend noch: Von fünf Jahre alten Fahrzeugen, die in die Prüfgasse fuhren, hatten 11,5\% einen Mangel an der Lichtanlage, bei sieben Jahren waren es 15,8\%, bei neun Jahren 21,6 \% - sogar jeder Vierte $(24,9 \%)$ ist es bei elf Jahren.

Doch ist auch eine positive Tendenz erkennbar: Dafür sorgen modernere Halogenlampen, Xenon-Licht und bald auch LEDs, die weniger anfällig bei Spannungsschwankungen sind und länger halten. Die Verbreitung der OnBoard-Diagnose führt laut TÜV dazu, dass Ausfälle früher bemerkt werden. Doch warum wird nicht gleich ein Lichtkontroll-Symbol im Cockpit zur Pflicht? Der Zustand des Drei-Wege-Kats und des Reifendrucks hat es doch auch ins Kombiinstrument geschafft.

Im Titelthema dieses Hefts stellen wir neue LED-Lichtsysteme von Audi und Hella vor. Ich bin gespannt, wie diese Innovation den Lichttest in elf Jahren beeinflusst haben wird.

Herzlichst, Ihr

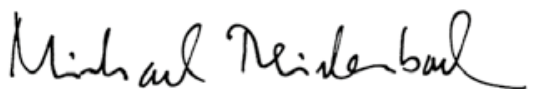

DIPL.-ING. MICHAEL REICHENBACH, Stellvertretender Chefredakteur Wiesbaden, 28. Januar 2014

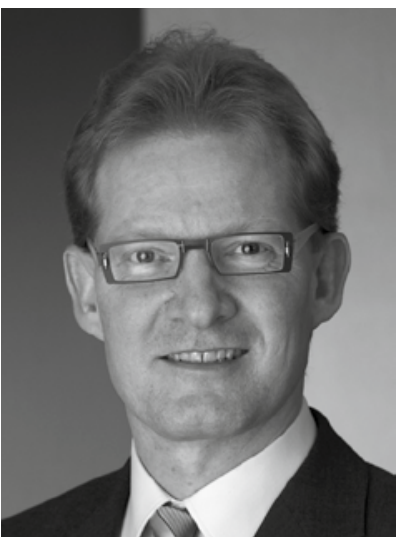

Wir

\section{entwickeln,} was bewegt

IAV - Ihr Partner

für Automotive Engineering 